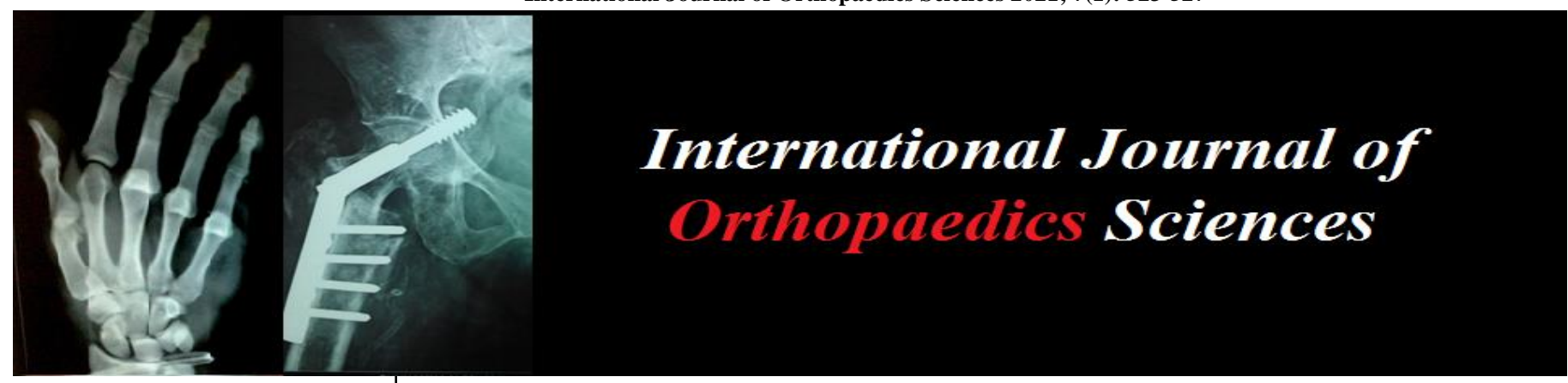

E-ISSN: 2395-1958

P-ISSN: 2706-6630

IJOS 2021; 7(1): 523-527

(C) 2021 IJOS

www.orthopaper.com

Received: 09-10-2020

Accepted: 18-11-2020

\section{Dosmanta Kutum}

Registrar, Department of

Orthopaedics \& Trauma,

Gauhati Medical College and

Hospital, Guwahati, Assam,

India

\section{Dr. Paragjyoti Gogoi}

Assistant Professor, Department

of Orthopaedics \& Trauma,

Gauhati Medical College and

Hospital, Guwahati, Assam,

India

\section{Mahibul Islam}

Assistant Professor, Department of Orthopaedics \& Trauma, Gauhati Medical College and Hospital, Guwahati, Assam, India

Partha Chakrabarty

Professor, Department of Orthopaedics \& Trauma,

Gauhati Medical College and Hospital, Guwahati, Assam, India
Corresponding Author: Dr. Paragjyoti Gogoi Assistant Professor, Department of Orthopaedics \& Trauma, Gauhati Medical College and Hospital, Guwahati, Assam, India

\section{Bone-patellar tendon-bone graft versus hamstring Tendon graft for arthroscopic complete ACL tear reconstruction: A randomized control study}

\author{
Dosmanta Kutum, Dr. Paragjyoti Gogoi, Mahibul Islam and Partha \\ Chakrabarty
}

DOI: https://doi.org/10.22271/ortho.2021.v7.i1i.2534

\section{Abstract}

Anterior Cruciate Ligament rupture is a common sports injury. The present treatment protocol is to reconstruct the ligament using autografts like bone-patellar tendon-bone, hamstring tendons, quadriceps tendon, peroneus longus tendon etc. Out of all these, the patellar tendon autograft and the quadrupled hamstring autograft are most commonly used. We have conducted a prospective randomised study comparing the outcome of anterior cruciate ligament reconstruction using these two autografts. A total of 40 patients were randomised into two groups with 20 patients each in the patellar tendon and hamstring group. Similar postoperative rehabilitation protocol was followed for each group and the final outcome was measured at the end of 6 months using Tegner and Lysholm score. The functional outcome of both the groups was statistically compared to find any significant advantage of one group over the other. The results we found were equivocal for both the groups. The return to preinjury status was also similar in both the groups.

Keywords: ACL reconstruction, patellar tendon, hamstring tendon, autograft, lysholm score

\section{Introduction}

The ACL functions as primary stabilizer for anterior translation of the tibia on the femur. It also acts in counteracting rotation and valgus stress. Anterior cruciate ligament deficiency or rupture leads to knee instability ${ }^{[1]}$. The frequent giving way symptoms in daily and sports activities leads to increased risk of meniscal injuries and early degeneration of the injured knee ${ }^{[2]}$. To counteract these, it is important to reconstruct the ligament; arthroscopic methods are now a day the gold standards. During the past decade, arthroscopic techniques evolved and refined for the reconstruction of the anterior and posterior cruciate ligaments ${ }^{[3]}$.

Anterior cruciate Ligament (ACL) tear is a commonly occurring ligamentous injury of the knee joint The graft used for reconstruction and the fixation techniques used varies among different surgeons ${ }^{[4]}$. The two most commonly used grafts for reconstruction are: Hamstring tendon \& Bone Patellar Bone tendon (BTB) graft. Most surgeons now prefer the patellar tendon autograft or hamstring tendons. For the past three decades, patellar tendon graft from the middle third of the patella tendon was most commonly used. Presently however, the combined semitendinosus and gracilis tendons (ST) graft exceeds it to avoid the complications [5]. Patellar tendon harvesting may lead to patellofemoral joint pain, patella fracture, patella tendon rupture, and infra patella contraction ${ }^{[6]}$. There are some complications also exist with the hamstring techniques. Most commonly, tunnel widening and loosening of fixation are found to affect the overall outcome along with other problems like saphenous nerve injury, hematoma etc. ${ }^{[7]}$. Multiple studies have published the long-term results of ACL reconstructions; however still it is inconclusive which one is better ${ }^{[8]}$.

We conducted a prospective, randomized clinical trial with an aim to compare bone-patellar tendon-bone (BPTB) grafts and four-strand semitendinosus-gracilis grafts for ACL reconstruction. Both groups were compared at one-year interval and the outcome measures consisted of return to pre-injury level of sporting activity, pain, knee stability, range of motion, IKDC (International Knee Documentation Committee) Score and complications. 


\section{Materials and Methods}

Between $1^{\text {st }}$ June 2018 and 31st may 2019, 40 patients (38 men and 02 women) with ACL tearing were selected for the study. Exclusion criteria were previous injury or operation on the knee, any fracture, osteoarthritis in either knee, or injury to other ligaments like posterior cruciate ligament, medial collateral ligament, lateral collateral ligament, and posterolateral complex. The study design was approved by the Ethics Committee of the Institute and all patients gave informed consent prior to inclusion in this trial. The patients were randomized by a computer-generated list into two groups, by two different treatment methods. The first group (Group $\mathrm{A} ; \mathrm{n}=20$ ) was treated with bone patellar bone autograft (BPTB); while in the second group (Group B; $n=$ 20) a Semitendinosus-Gracilis graft (ST) was used.

\section{Surgical procedure}

The surgery was performed under spinal anaesthesia and the tourniquet was used in all patients. The knee was thoroughly examined for the laxity by drawers and Lachman test and varus-valgus stress. Pivot shifting was also elicited as well. After preparing and drapping, an arthroscopic evaluation of the joint was done and the diagnosis of ACL tear confirmed; and the associated meniscal tear addressed. After that the autograft of Central third patellar tendon or hamstring tendons harvested according to the randomisation for that particular patient. The patellar tendon was exposed by a single incision and with the help of small saw and fine osteotome the graft is removed with bone plugs [Figure 1]. Both the bone ends were tagged with No 5 ethibond suture, length measured and kept safely. In case of hamstring autograft, first the Semitendinosus was harvested, length measured, quadrupled and the diameter assessed. If inadequate then the Gracilis was also harvested. Then arthroscopically the femoral and tibial tunnels were prepared. We used the transportal method for preparation of the femoral tunnel using appropriate guide. The tibial tunnel was made using tibial guide zig with elbow pointer. In case of bone-patellar tendon-bone autograft both femoral and tibial sides were fixed by titanium interference screws of proper size. In case of hamstring tendon autograft, the femoral side was fixed with endobutton with fixed or variable loop and the tibial side with Bioscrews of appropriate size. The wound was closed and the knee was immobilised with a brace.

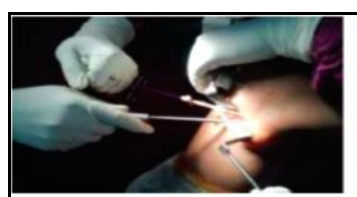

A

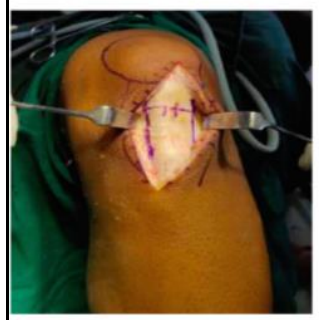

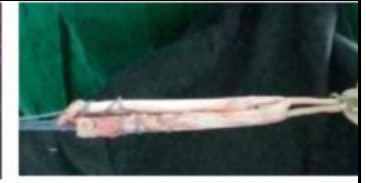

B

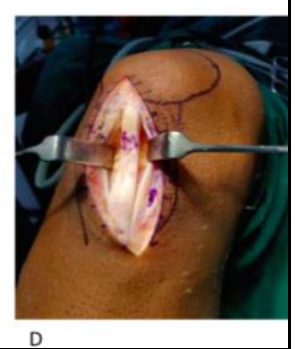

Fig 1: A \& B-Hamstring Graft harvesting; C\&D-Patellar Tendon harvesting

\section{Rehabilitation protocol}

The knee was immobilised for two weeks in a brace. During this period the patient was encouraged to continue quadriceps exercises, ankle movements and calf muscle exercises. Walking allowed once the pain was gone, usually after two days. Stitches were removed after ten days and knee bending started with a stop at 45 degrees. After four weeks the kneebending angle was increased with an aim to get 90 degree bending at six weeks [Figure $2 \& 3$ ] Hamstring strengthening exercises were started at the same time of knee bending. After twelve weeks brisk walking allowed and after sixteen weeks straight line jogging started. Cutting sports were allowed after six months. Final evaluation of the knee was done at six weeks in regards of laxity and Lysholms functional scores.
A

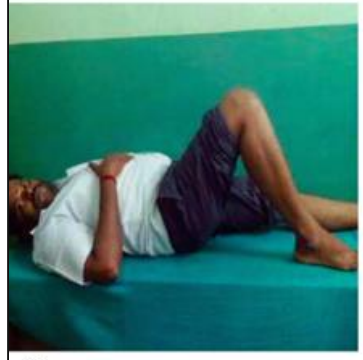

C

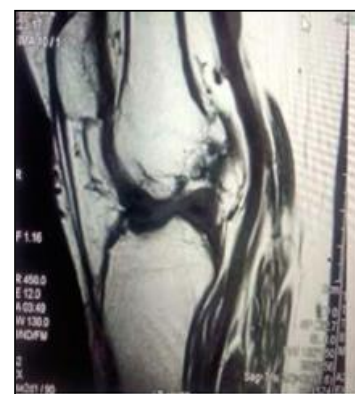

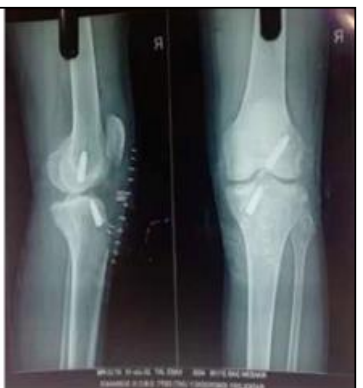

B
Fig 2: A-MRI of the Knee with torn ACL, B-Post operative X-ray of the Knee after BTB autograft, C-ROM of the Knee at 6 months

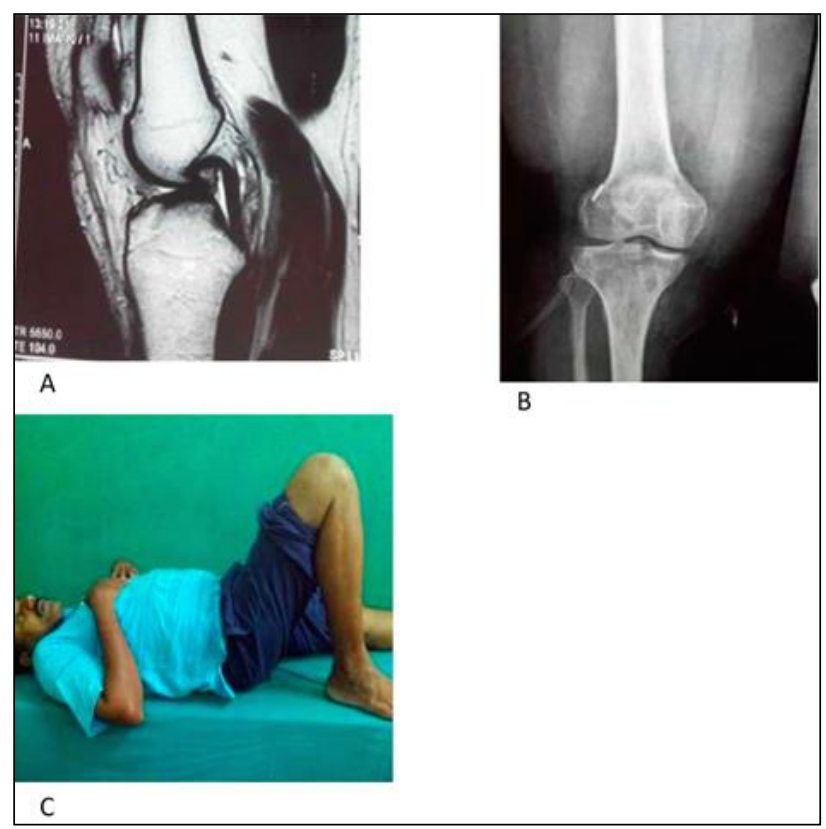

Fig 3: A-MRI Knee with torn ACL, B-Hamstring autograft fixed with Endobutton \& Bioscrew, C-ROM of the Knee at 6 months 


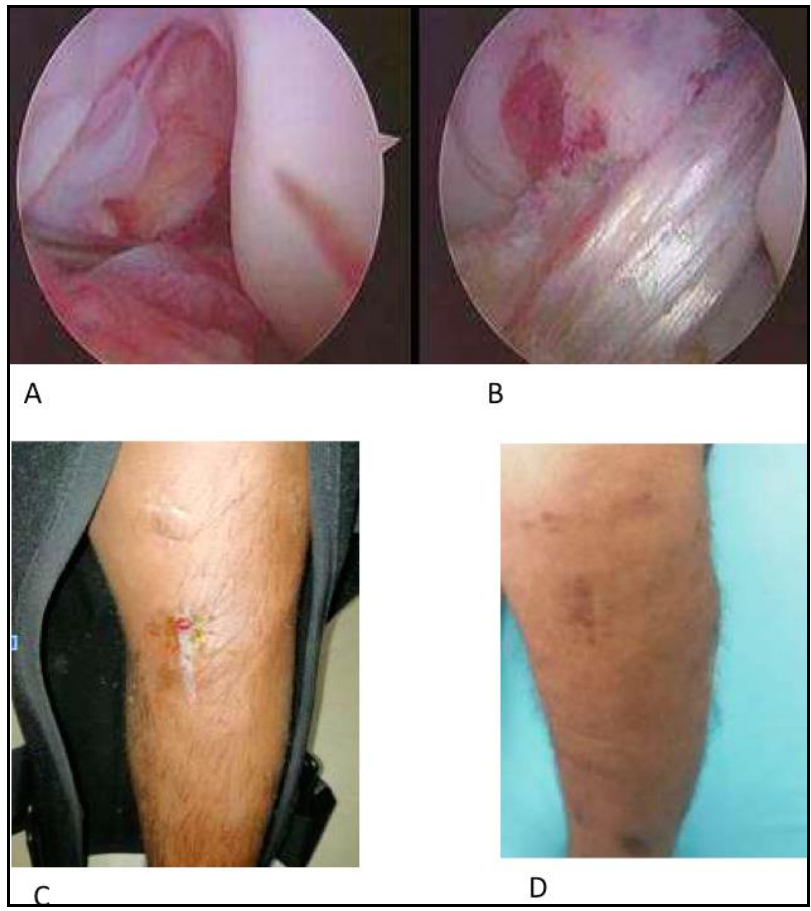

Fig 4: A\&B-Arthroscopic picture of torn and Reconstructed ACL, C\&D-Superficial Infection which healed conservatively

\section{Results}

There were no significant differences between the two groups in the findings of meniscal or osteochondral lesions. At the 12 month follow-up 14 patients $(70 \%)$ in group A (BPTB) and 15 patients $(75 \%)$ in group B had good-to-excellent IKDC score (grade A or B), showing statistically insignificant differences between the two group ( $p>0.05)$, (Table 1). The activity levels as measured with the Tegner scale at the one year follow-up was a mean of 6 points (range, 3 to 9 points) in the BPTB group and a mean of 5 points (range, 4 to 9 points) in the Hamstring group $(\mathrm{p}>0.05)$. Postoperatively for Lachman test, In BPTB group, 13 patients were graded as normal, 5 patients were graded as $1+$ and 2 patients as $2+$ and in the ST group 11 patients were graded as normal, 7 patients graded as $1+$ and 2 patients graded as +2 . The differences were statistically insignificant $(\mathrm{p}>0.05)$.

Regarding the Pivot Shift test, there was a statistically significant improvement in the integrity of the ACL in both the groups, and no significant difference was noted between the two groups $(p>0.05)$. No significant differences noted in parameters like Thigh Circumference difference, Effusion, or Range of motion between the two groups. Greater number of our patients was seen in the age group of 26-30 yrs. Male preponderance was noticed in our study. Left side was involved more commonly than the right side.

Road traffic accident was the most common cause accounting for ACL injury. (Table 2) In relation to the associated conditions, the Medial meniscus injury was involved more than the lateral meniscus. The average age of the Patients was 28.87 years (range; $20-40$ years). The time between injury and surgery ranged from 6 weeks to 12 months, and it was similar for the two groups.

Table 1: Outcome measures using Lysholm and Gillquist scoring method

\begin{tabular}{|c|c|c|c|c|}
\hline Outcome & Bone patella tendon bone graft GP. no of patients (20) & Percentage & Hamstring auto graft GP. No of patients (20) & Percentage \\
\hline Excellent & 6 & 30 & 7 & 35 \\
\hline Good & 8 & 40 & 8 & 40 \\
\hline Fair & 6 & 30 & 5 & 25 \\
\hline Poor & 0 & 0 & 0 & 0 \\
\hline
\end{tabular}

By using Chi Square test, $\mathrm{P}=0.1678$

Table 2: Associated Injury along with ACL Tear

\begin{tabular}{|c|c|c|}
\hline Associated injury & Patients & Percentage \\
\hline Medial Meniscus Tear & 16 & 40 \\
\hline Lateral Meniscus Tear & 4 & 10 \\
\hline Both & 2 & 5 \\
\hline Nil & 18 & 45 \\
\hline Total & 40 & 100 \\
\hline
\end{tabular}

\section{Discussion}

The BPTB and four-strand hamstring grafts (4SHG) are the most common currently used grafts for ACL reconstruction. Controversy is still there over which one gives the best results. The aim of this article was to compare the effectiveness of these two autografts by comparing the results of specific outcomes in 40 patients. Of the earlier prospective studies comparing BPTB and hamstring grafts, some showed the graft materials to have similar laxity values, and others showed significantly better values for the BPTB grafts. In this study the outcome of BPTB reconstructions compared with quadrupled hamstring reconstructions showed equal stability in treatment of ACL tearing. Both group of patients had IKDC scores comparable with previous studies.

In 2002, Beynnon et al. conducted a prospective study of 68 patients of ACL reconstruction using either a two-strand semitendinosus autograft or a BPTB autograft and assessing knee function and IKDC score at 3-year follow-up ${ }^{[9]}$. They found good-to-excellent IKDC scores in $82 \%$ and $86 \%$ of patients having patella-tendon and hamstring reconstructions, respectively. It is in accordance with our series that showed good-to-excellent scores in $70 \%$ and $75 \%$ of the patellatendon and semitendinosus group, respectively, at 1-year follow up.

The IKDC score comparison between the groups along with ligament laxity, similar outcomes were noted. In our study, the patella tendon group had similar postoperative Tegner scores than the hamstring group, which reflects a return to higher activity level; however, some studies showed that KT1000 arthrometer side-to-side differences and objective measurement of knee stability are not directly correlated with knee outcome scores [10,11, 12, 13]. Extension loss was more common in the group treated with BPTB graft and flexion loss was more commonly seen in patients treated with hamstring graft ${ }^{[14]}$. Like the previous studies, we found that the number of patients with extension loss was more in patellar tendon graft group and the number of patients with flexion loss was more in hamstring graft group. However, our results did not reach statistical significance.

In our trial both treatments resulted in similar outcomes with regard to prevalence of knee-locking, ability to weight bearing, squatting, climbing stairs, running and thigh circumference difference. Otero et al. noted that although patellofemoral crepitus was more common after BPTB reconstructions (29\% in comparison with $19 \%$ after hamstring reconstruction), anterior knee pain was nearly three times 
more common in the hamstring group ${ }^{[15]}$. It was in contrary to Corry et al. who found $31 \%$ of their BPTB group had kneeling pain after 2 years ${ }^{[16]}$. The incidence in the hamstring group was only $6 \%$ after 2 years, and this was statistically significant. However, there was no any statistically significant difference noted for patellofemoral pain between the two groups. Neither group in our study underwent an aggressive postoperative rehabilitation protocol nor running delayed until 3 months postoperatively. These provide adequate time for the hamstring graft to heal within bony tunnels. The BPTB graft usually heals by bone-to-bone in around 6 to 8 weeks. The 4SHG graft does heal to bone via Sharpey's like fibers, but this takes around 12 weeks ${ }^{[17]}$. In order to allow unrestricted mobilization, the graft fixation must be able to at least withstand the normal forces in the native ACL (approximately $2500 \mathrm{~N}$ ). Blickenstaff et al. and Scranton et al. suggested to avoid overloading of hamstring grafts during healing $[18,19]$. Therefore, it is assumed that the conservative rehabilitation program may adversely affected the outcome of the hamstring tendon group.

Two-strand hamstring grafts are no longer used now as found an inadequate graft and a potential cause of poor functional outcome. The semitendinosus-gracilis graft technique used in this study was quadruple-looped autograft and although it has stiffness comparable to the natural ACL and to patellar tendon autografts, the patella tendon group in our study had better laxity values. We assume that it is due to different graft fixation techniques. Furthermore, the more rapid healing of the bone plugs into the graft tunnels may account in part for the less failure rates observed in the patellar tendon group. Apart from graft tear midway, the main concern during hamstring graft harvest is the inconsistency of the graft in length and thickness ${ }^{[19]}$. Congenitally small tendons may not be a suitable graft. A nearly avulsed tendon may also be too short to use. Other problems to surgeons using the hamstring graft include the gradually increasing anterior laxity, the longer healing time and graft fixation failures [Figure 4].

\section{Conclusion}

The results of our study were comparable with already published reports of comparative study done using bone patellar tendon bone versus hamstring graft. Our study shows that there is no difference in functional outcome whether bone patella tendon bone graft or hamstring autograft was used. The success of ACL reconstruction depends on the correct technique used for the surgery, precise placement of graft and proper-guided rehabilitation methods.

\section{Conflict of Interest: None.}

Fund Received for the Study: None.

\section{References}

1. Butler DL, Noyes FR, Grood ES. Ligamentous restraints to anterior-posterior drawer in the human knee. J Bone Joint Surg Am 1980;62(2):259-70.

2. Haimes JL, Wroble RR, Grood ES, Noyes FR. Role of the medial structures in the intact and anterior cruciate ligament-deficient knee: limits of motion in the human knee. The American journal of sports medicine 1994;22(3):402-9.

3. Fu FH, Bennett CH, Ma CB, Menetrey J, Lattermann C. Current trends in anterior cruciate ligament reconstruction: Part II. Operative procedures and clinical correlations. The American journal of sports medicine 2000;28(1):124-30.

4. Kurosaka M, Yoshiya S, Andrish JT. A biomechanical comparison of different surgical techniques of graft fixation in anterior cruciate ligament reconstruction. The American journal of sports medicine 1987;15(3):225-9.

5. Hamner DL, Brown CH, Steiner ME, Hecker AT, Hayes WC. Hamstring tendon grafts for reconstruction of the anterior cruciate ligament: biomechanical evaluation of the use of multiple strands and tensioning techniques. JBJS 1999;81(4):549-7.

6. Rubinstein JR RA, Shelbourne KD, Van Meter CD, McCarroll JC, Rettig AC. Isolated autogenous bonepatellar tendon-bone graft site morbidity. The American journal of sports medicine 1994;22(3):324-7.

7. Friedman MJ. Arthroscopic semitendinosus (gracilis) reconstruction for anterior cruciate ligament deficiency. Techniques in orthopaedics 1988;2(4):74-80.

8. Holm I, Øiestad BE, Risberg MA, Aune AK. No Difference in Knee Function or Prevalence of Osteoarthritis after Reconstruction of the Anterior Cruciate Ligament with 4-Strand Hamstring Autograft versus Patellar Tendon-Bone Autograft: A Randomized Study with 10-Year Follow-up. The American journal of sports medicine 2010;38(3):448-54.

9. Beynnon BD, Johnson RJ, Fleming BC, Kannus P, Kaplan M, Samani J et al. Anterior cruciate ligament replacement: comparison of bone-patellar tendon-bone graft with two strand hamstring graft. A prospective, randomized study. J Bone Joint Surg (Am) 2002;84-A:1503-13.

10. Aune AK, Holm I, Risberg MA, Jensen HK, Steen H. Four-strand hamstring tendon autograft compared with patellar tendon-bone autograft for anterior cruciate ligament reconstruction: a randomized study with twoyear follow-up. The American journal of sports medicine 2001;29(6):722-8.

11. Wagner M, Kääb MJ, Schallock J, Haas NP, Weiler A. Hamstring tendon versus patellar tendon anterior cruciate ligament reconstruction using biodegradable interference fit fixation: a prospective matched-group analysis. The American journal of sports medicine 2005;33(9):1327-36.

12. Gulick DT, Yoder HN. Anterior cruciate ligament reconstruction: clinical outcomes of patella tendon and hamstring tendon grafts. Journal of sports science \& medicine 2002;1(3):63.

13. Steiner ME, Hecker AT, Brown $\mathrm{Jr} \mathrm{CH}$, Hayes WC. Anterior cruciate ligament graft fixation: comparison of hamstring and patellar tendon grafts. The American journal of sports medicine 1994;22(2):240-7.

14. Rosenberg TD, Franklin JL, Baldwin GN, Nelson KA. Extensor mechanism function after patellar tendon graft harvest for anterior cruciate ligament reconstruction. The American journal of sports medicine 1992;20(5):519-26.

15. Otero AL, Hutcheson L. A comparison of the double semitendinosus/gracilis and central third of the patellar tendon autografts in arthroscopic anterior cruciate ligament reconstruction. Arthroscopy 1993; 9:143-8.

16. Corry IS, Webb JM, Clingeleffer AJ, Pinczewski LA. Arthroscopic reconstruction of the anterior cruciate ligament. A comparison of patellar tendon autograft and four stranded hamstring tendon autograft. Am J Sports Med 1999;27:444-54.

17. Abe S, Kurosaka M, Iguchi T, Yoshiya S, Hirohata K. Light and electron microscopic study of remodeling and maturation process in autogenous graft for anterior cruciate ligament reconstruction. Arthroscopy: The Journal of Arthroscopic \& Related Surgery 1993;9(4):394405. 
18. Blickenstaff KR, Grana WA, Egle D. Analysis of a semitendinosus autograft in a rabbit model. Am J Sports Med 1997;25:554-9.

19. Scranton PE Jr, Lanzer WL, Ferguson MS, Kirkman TR, Pflaster DS. Mechanisms of anterior cruciate ligament neovascularization and ligamentization. Arthroscopy 1998;14:702-16.

20. Marx RG, Spock CR. Complications following hamstring anterior cruciate ligament reconstruction with femoral cross-pin fixation. Arthroscopy: The Journal of Arthroscopic \& Related Surgery 2005;21(6):762. 